\title{
Effects of Angiotensin-Converting Enzyme Inhibitors on Arterial Stiffness: A Systematic Review and Meta-Analysis of Randomized Controlled Trials
}

\author{
Xiuli Li, Peng Chang, Qiongying Wang (D), Hao Hu, Feng Bai, Ningyin Li, and Jing Yu (iD \\ Department of Cardiology, Lanzhou University Second Hospital, Lanzhou, China \\ Correspondence should be addressed to Jing Yu; yujing2304@163.com
}

Received 4 October 2019; Revised 31 December 2019; Accepted 8 January 2020; Published 1 March 2020

Academic Editor: Kailash Prasad

Copyright ( 2020 Xiuli Li et al. This is an open access article distributed under the Creative Commons Attribution License, which permits unrestricted use, distribution, and reproduction in any medium, provided the original work is properly cited.

\begin{abstract}
To determine the effects of ACEIs on arterial stiffness, a meta-analysis of randomized controlled trials was conducted. Relevant articles that investigated the effects of ACEIs on arterial stiffness from PubMed, Embase, and the Cochrane library from inception to September 2018 were systematically retrieved. The investigated outcomes included brachial-ankle pulse wave velocity (baPWV) and carotid-femoral PWV (cf-PWV) by using weighted mean differences (WMDs) and 95\% confidence intervals (CIs) with the random-effects model. A total of 17 RCTs including 1,458 individuals were included. The summary results indicated no significant differences between ACEIs and control for ba-PWV and cf-PWV. Also, no significant differences between ACEI and control for ba-PWV and cf-PWV were observed in hypertensive patients, while the therapeutic effects of ACEI versus placebo showed statistically significant difference. Moreover, subgroup analysis indicated that the levels of ba-PWV were significantly associated if the study was conducted in Western countries, mean age $<60.0$ years, percentage male $\geq 60.0 \%$, compared with ARBs, baseline PWV <10.0, and high-quality study. Furthermore, the significant levels of cf-PWV in patients who received ACEIs were observed when percentage male was $\geq 60.0 \%$ and the studies were of high-quality. Finally, no significant differences were observed between ACEIs and other antihypertensive drugs regarding the changes of systolic blood pressure (SBP) and diastolic blood pressure (DBP). The overall analysis suggested no significant differences between ACEIs and other antihypertensive drugs for baPWV and cf-PWV levels, whereas ACEIs versus placebo showed lower levels of ba-PWV and cf-PWV.
\end{abstract}

\section{Introduction}

Cardiovascular disease is one of the major diseases that seriously threaten the health of several people worldwide [1]. Currently, there are several studies that have illustrated the pathological changes of the vascular wall that underlie cardiovascular events and play an important role in the progression of cardiovascular diseases [2-4]. Furthermore, changes in the structure of arterial wall caused declination in arterial compliance that might precede the clinical symptoms of the disease [5-7]. Therefore, arterial elasticity and function are used as a variety of cardiovascular risk factors for subclinical vascular lesions [8-10]. Recently, it has been demonstrated that arterial stiffness is another important risk factor that is independent of other cardiovascular risk factors and is considered to be an alternative endpoint of cardiovascular disease, and is associated with the morbidity and mortality of cardiovascular disease [11-13].

According to a previous study, angiotensin II is a potent vasoactive peptide in endothelial renin-angiotensin system (RAS), and angiotensin receptor blockers lower blood pressure (BP) and improve arterial elasticity [14]. Therefore, we speculated the beneficial impact of angiotensin-converting enzyme inhibitors (ACEIs) on arterial stiffness that extends beyond BP reduction [15]. Many studies illustrated the potential impact of ACEIs on arterial stiffness in various populations, and reported a protective role of ACEIs against arterial stiffness in hypertensive patients [16, 17]. Further, hypertension is characterized by thickened arterial wall, reduced blood vessel elasticity, and lumen dilatation prior to the rise in BP, which involves as vascular remodeling and is also a major cause of hypertensive complications. The 
structure and function of blood vessels should be restored in hypertensive patients receiving ACEIs, reducing the occurrence of cardiovascular and cerebrovascular events [18-21].

Arterial stiffness is predominantly reflected by the traveling speed of this pulse wave, and is termed as the pulse wave velocity (PWV) [22, 23]. According to the Europe Hypertension Management Guidelines that was published by the Society of Hypertension and the European Society of Cardiology (ESC/ESH) for the first time, PWV is included as one of the limb indicators for assessing subclinical target organ damage in hypertension [24]. Studies have emphasized the importance of applying PWV for representing arterial stiffness [8, 25-27]. Mallareddy et al. evaluated adult hypertensive patients without complex arterial structural or hemodynamic changes administered an ACEI for $>4$ weeks, and confirmed that ACEIs have a role in reducing PWV levels [28]. Meanwhile, Kithas and Supiano evaluated patients with essential hypertension otherwise in good general health (no underweight or morbid obesity) after 6 months of treatment, and confirmed the role of hydrochlorothiazide and spironolactone in lowering PWV levels [29]. However, there is a lack of latest meta-analysis results regarding the comparison of the effects of ACEIs versus placebo or other hypertensive agents on PWV (brachial-ankle PWV [baPWV] and carotid-femoral PWV [cf-PWV]) levels. Therefore, this meta-analysis was performed to assess the effectiveness of ACEIs on arterial stiffness.

\section{Methods}

2.1. Data Sources, Search Strategy, and Selection Criteria. This review was conducted and reported according to the guidelines of Preferred Reporting Items for Systematic Reviews and Meta-Analysis Statement issued in 2009 (Checklist S1) [30]. RCTs published in English and those that investigated the effectiveness of ACEIs on arterial stiffness were systematically searched from PubMed, Embase, and the Cochrane library till September 2018. There were no restrictions placed on publication status (published, in press, or in progress). The main search terms used were as follows: ("ramipril" or "cilazapril" or "quinapril" or "perindopril" or "lisinopril" or "captopril" or "Temocapril" or "quinapril" or “angiotensin-converting enzyme inhibitors" or "ACEI”) AND ("pulse wave velocity" or "PWV") and are defined as "humans," and "randomized controlled trials." Furthermore, the references of eligible studies were manually searched for any eligible articles.

The literature search and study selection were undertaken by 2 authors independently by using a standardized approach. Any inconsistencies were settled by a corresponding author. Studies were considered eligible for inclusion if the following criteria were met: (1) studies with RCT design; (2) adult patients regardless of disease status; (3) the intervention was ACEIs and the control group was placebo or other hypertensive agents; and (4) the study should report PWV (ba-PWV and cf-PWV). The exclusion criteria were as follows: animal experiments and duplicated studies; studies designed as cross-over designs; and PWV was not categorized as ba- or cf-PWV.
2.2. Data Collection and Quality Assessment. All reliable and interested data of baseline characteristics and primary endpoints in the studies are extracted by two reviewers, respectively. In addition, if more than two arms compared the efficacy of ACEIs vs. other medications, all the relevant data associated with other medications should be pooled and interested endpoints were extracted and used to perform the pooled analysis. The data collected included the following information: first author's name, publication year, country, sample size, mean age, percentage male, baseline systolic blood pressure/diastolic blood pressure (SBP/DBP), intervention, control, follow-up duration, baseline PWV, and the PWV and SBP/DBP levels after interventions. The Jadad score was used to evaluate methodological quality of RCTs in this meta-analysis [31]. Briefly, the overall scale of a clinical trial was described from 0 to 5 if using the Jadad scale. In this meta-analysis, individual trials with scores 4 or 5 are regarded as high-quality studies.

2.3. Statistical Analysis. Continuous variables were presented as mean and standard deviation, the summary of weighted mean differences (WMDs) and 95\% confidence intervals (CIs) were employed to calculate the effect of ACEIs on arterial stiffness, and SBP/DBP was evaluated by using random-effects model $[32,33]$. The heterogeneity size was determined with $I^{2}$ and $Q$ statistics, and $P$ value of less than 0.10 was considered as significant heterogeneity [34, 35]. Sensitivity analysis was conducted for investigating the outcomes to evaluate the impact of single study on overall analysis [36]. Subgroup analyses were conducted for ba-PWV, cf-PWV, SBP, and DBP based on country, mean age, percentage male, follow-up duration, baseline PWV, and study quality after excluding the study that included patients with normal blood pressure (mean $\mathrm{SBP}<140 \mathrm{~mm} \mathrm{Hg}$ ). Furthermore, interaction tests were performed to evaluate the differences between subgroups [37]. Funnel plots were drawn, and were used to perform Egger and Begg tests for the included articles for determining the presence of any publication bias [38, 39]. The test level for pooled results, sensitivity, subgroup, and publication bias was 0.05 . Statistical analyses were performed by using STATA software (version 10.0; Stata Corporation, College Station, TX, USA).

\section{Results}

3.1. Literature Selection. The search results of RCTs by the two reviewers were highly consistent. After utilizing the search strategy, a total of 513 published articles were retrieved. After screening the titles and abstracts according to the inclusion and exclusion criteria, 438 articles were discarded due to irrelevance of topic, animal studies, drug treatment, or improper evaluation of effectiveness and outcomes. Reading the full texts of the remaining 75 studies yielded 17 studies for inclusion, and 58 studies were excluded due to non-RCT or cross-over designs, no desired outcomes (ba-PWV and cf-PWV), or no appropriate control. Finally, 17 studies were selected for final meta-analysis 
[40-56]. The results of study-selection process are presented in Figure 1.

3.2. Characteristics of Included Studies. The general characteristics of each included study are listed in Table 1. There were 1,458 patients in the included RCTs, and their mean age ranged from 32.8 to 69.0 years. The included studies were conducted in Argentina, Japan, Poland, Malaysia, UK, Italy, Greece, and Spain. Among the included studies, 4 studies reported ba-PWV and 14 studies reported cf-PWV. Fifteen studies included hypertensive patients, and the remaining 2 studies included individuals with normal BP. The duration of intervention ranged from 8-48 weeks. Study quality was evaluated by using the Jadad scale, and 9 studies had a score of 4 , and the remaining had a score of 3 .

3.3. $b a-P W V$. Data regarding the effect of ACEIs on baPWV were available from 4 studies. The summary results indicated no significant differences between ACEIs and control regarding the change of ba-PWV levels (WMD: -0.35 ; $95 \%$ CI: -2.06 to $1.36 ; P=0.688$; Figure $2(a)$ ), and significant heterogeneity was observed $(P<0.001)$. Furthermore, no significant differences were observed in hypertensive patients (WMD: 0.31 ; 95\% CI:-0.48 to 1.10 ; $P=0.443$; substantial heterogeneity), and ACEIs were associated with lower ba-PWV levels in normal subjects (WMD: -2.40 ; 95\% CI: -2.54 to -2.26 ; $P<0.001$ ). Hence, a sensitivity analysis was conducted, while the conclusion was unaffected by sequential exclusion of any specific study (Figure 2(b)). Although country, mean age, percentage male, control, baseline PWV, and study quality might affect the treatment effects of ACEIs on ba-PWV levels $(P<0.05)$, subgroup analyses indicated that the ACEIs were associated with increased levels of ba-PWV if the study was conducted in Western countries (WMD: 1.02; 95\% CI: 0.17 to 1.87 ; $P=0.019$ ), mean age of $<60.0$ years (WMD: $0.71 ; 95 \% \mathrm{CI}$ : 0.04 to $1.39 ; P=0.037$ ), percentage male $\geq 60.0 \%$ (WMD: 1.02; 95\% CI: 0.17 to $1.87 ; P=0.019$ ), compared with ARB (WMD: 0.71; 95\% CI: 0.04 to $1.39 ; P=0.037$ ), baseline PWV of $<10.0$ (WMD: 1.02 ; $95 \%$ CI: 0.17 to $1.87 ; P=0.019$ ), and high-quality studies (WMD: 1.02 ; $95 \%$ CI: 0.17 to 1.87 ; $P=0.019)$ (Table 2). The publication bias for ba-PWV was shown in Figure 2(c), and the Egger $(P=0.081)$ or Begg test $(P=1.000)$ results showed no significant publication biases for ba-PWV.

3.4. $c f-P W V$. Data regarding the effects of ACEIs on cfPWV were available from 14 studies. There was no significant difference between ACEIs and control for cf-PWV level (WMD: $-0.44 ; 95 \% \mathrm{CI}:-0.96$ to $0.09 ; P=0.104$; Figure $3(\mathrm{a})$ ). Heterogeneity was observed in the magnitude of the effect across the trials $(P<0.001)$. No significant difference was observed in hypertensive patients (WMD: -0.13 ; $95 \%$ CI: -0.54 to $0.27 ; P=0.528$; substantial heterogeneity), and ACEI was associated with lower cf-PWV levels in normal individuals (WMD: -2.05 ; $95 \%$ CI: -2.15 to -1.94; $P<0.001$; without evidence of heterogeneity). The

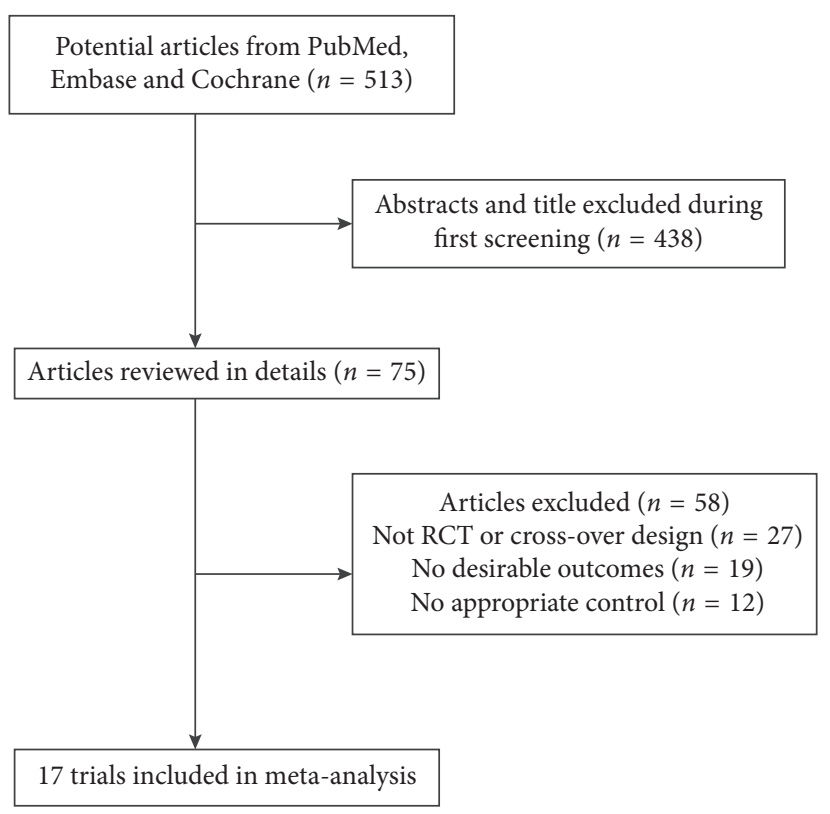

FIGURE 1: Flow chart of the study-selection process. Of the 513 hits retrieved in the initial database search, 438 were excluded by reviewing abstracts and titles, leaving 75 trials for assessment. After reviewing the full texts, 58 reports were excluded, and a total of 17 articles were included in this meta-analysis.

sensitivity analysis results indicated change in the pooled results after excluding the study conducted by Mackenzie et al., [51] which specifically included elderly patients (Figure 3(b)). Subgroup analysis indicated that the ACEIs were associated with lower levels of cf-PWV when the percentage male was $\geq 60.0 \%$ (WMD: -0.40 ; $95 \%$ CI: -0.75 to $-0.04 ; P=0.031)$ and studies with high-quality scores (WMD: $-0.41 ; 95 \%$ CI: -0.79 to $-0.03 ; P=0.032$ ) (Table 3). $P$ value for interaction tests indicated that country, mean age, percentage male, control, follow-up duration, baseline PWV, and study quality could bias the therapeutic effects between ACEI and control groups $(P<0.05)$. There was no significant publication bias for cf-PWV $(P$ value for Egger: 0.979; $P$ value for Begg: 0.827; Figure 3(c)).

3.5. SBP and DBP. All the included studies reported SBP and SBP at baseline and after interventions. The summary WMD indicated that patients who received ACEI showed no significant difference in SBP compared with the control group (WMD: -0.70 ; $95 \%$ CI: -2.72 to $1.33 ; P=0.500$; Figure 4(a)). Moreover, significant heterogeneity was observed among the included trials. Furthermore, no significant difference was observed between ACEI and control groups regarding the change of DBP (WMD: 0.26 ; $95 \%$ CI: -1.50 to $2.02 ; P=0.772$; Figure 4(b)), and substantial heterogeneity was detected. Subgroup analyses indicated that ACEI was associated with greater reduction in SBP when the follow-up duration was $>24.0$ weeks (WMD: -4.43 ; 95\% CI: -8.61 to -0.25 ; $P=0.038$ ), whereas ACEI was associated with higher SBP when the baseline PWV was $<10$ (WMD: 3.67; 95\% CI: 0.11 to 7.23; $P=0.043$; Table 4). Also, ACEI was associated with 


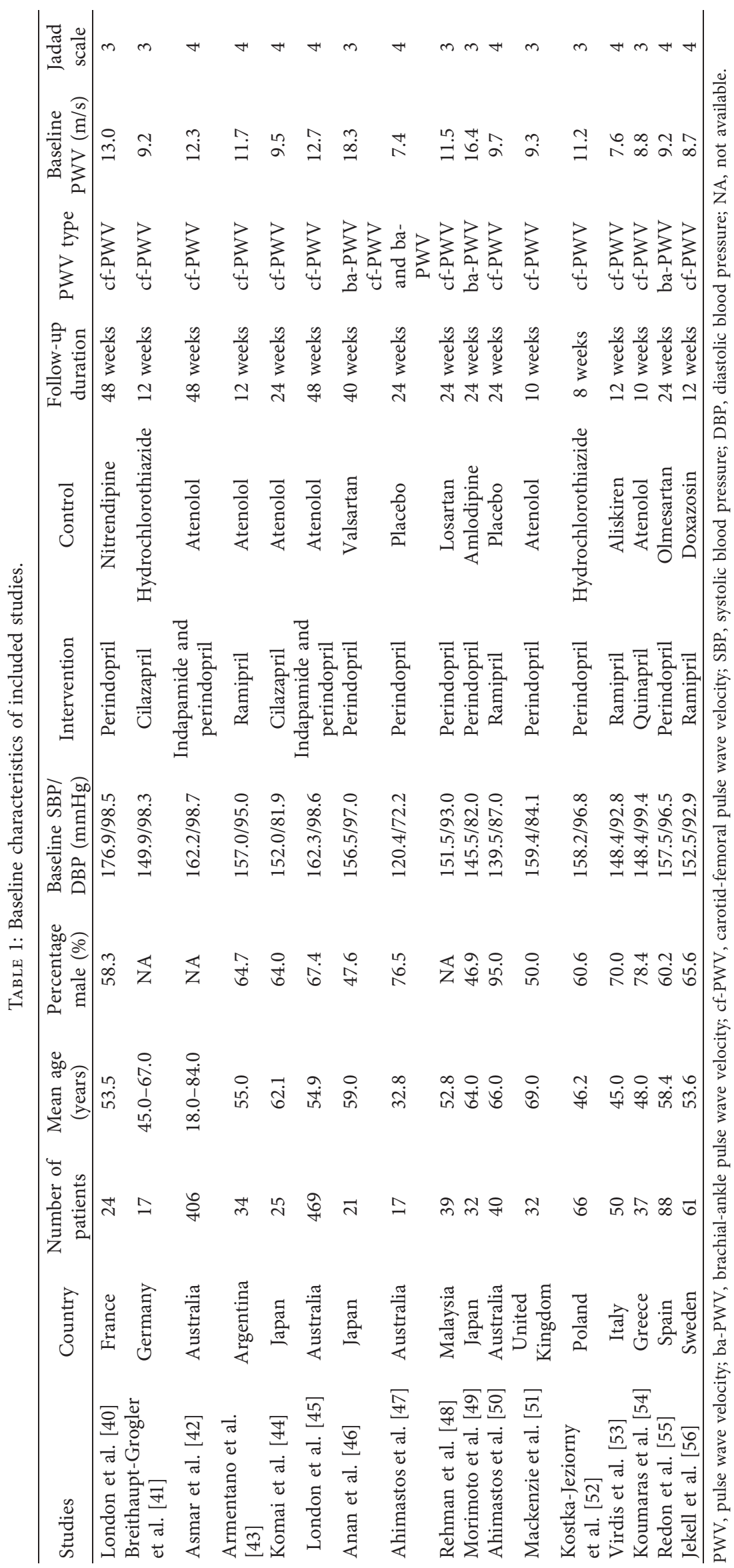




\begin{tabular}{|c|c|c|c|}
\hline Study & & $\begin{array}{l}\text { Mean difference } \\
\quad(95 \% \mathrm{CI})\end{array}$ & $\%$ weight \\
\hline Anan 2005 & & $0.33(-0.64,1.30)$ & 24.0 \\
\hline Ahimastos 2007 & & $-2.40(-2.54,-2.26)$ & 25.9 \\
\hline Morimoto 2008 & & $-0.22(-0.62,0.18)$ & 25.6 \\
\hline Redon 2016 & & $1.02(0.17,1.87)$ & 24.4 \\
\hline Overall & & $\begin{array}{c}-0.35(-2.06,1.36) ; P=0.688 \\
(I \text {-square: } 98.3 \% ; P<0.001)\end{array}$ & 100.0 \\
\hline-5 & $\begin{array}{lll}-2 & 0 & 2\end{array}$ & 5 & \\
\hline
\end{tabular}

(a)

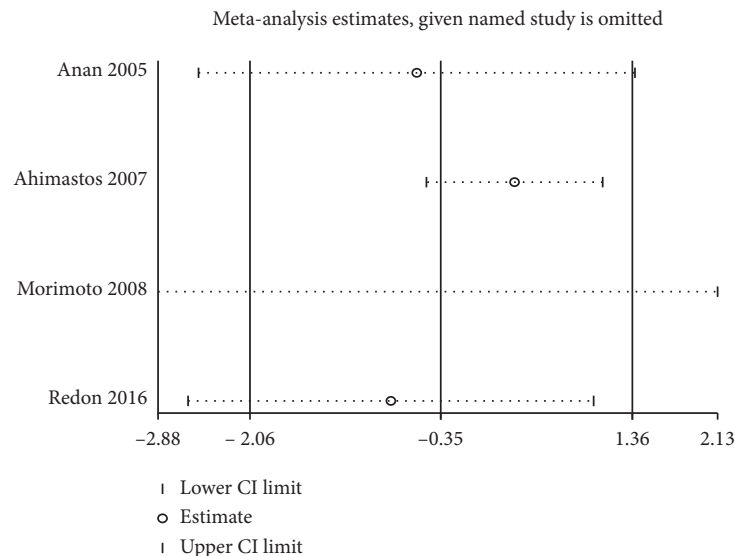

(b)

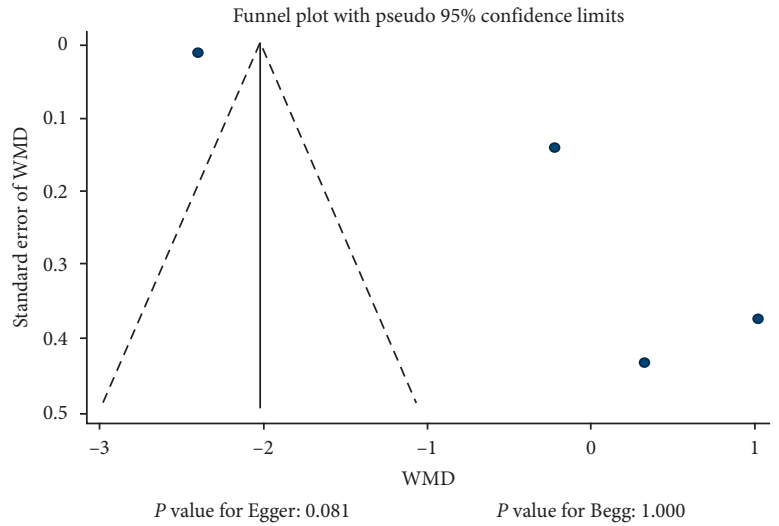

(c)

FIGURE 2: Effects of ACEIs on ba-PWV. (a) Summary of mean differences; (b) sensitivity analysis; (c) funnel plot for publication bias assessment, by Egger's and Begg's methods. CI, confidence interval; WMD, weighted mean difference; ACEIs, angiotensin-converting enzyme inhibitors; ba-PWV, brachial-ankle pulse wave velocity.

TABLE 2: Subgroup analyses for ba-PWV.

\begin{tabular}{|c|c|c|c|c|c|}
\hline Group & WMD and $95 \% \mathrm{CI}$ & $P$ value & Heterogeneity (\%) & $\begin{array}{c}P \text { value for } \\
\text { heterogeneity }\end{array}$ & $\begin{array}{c}P \text { value for } \\
\text { interaction test }\end{array}$ \\
\hline \multicolumn{6}{|l|}{ Country } \\
\hline Eastern & $-0.13(-0.53$ to 0.27$)$ & 0.527 & 6.1 & 0.302 & 0.014 \\
\hline Western & $1.02(0.17$ to 1.87$)$ & 0.019 & - & - & \\
\hline \multicolumn{6}{|c|}{ Mean age (years) } \\
\hline$\geq 60.0$ & $-0.22(-0.62$ to 0.18$)$ & 0.275 & - & - & 0.014 \\
\hline$<60.0$ & $0.71(0.04$ to 1.39$)$ & 0.037 & 9.3 & 0.294 & \\
\hline \multicolumn{6}{|c|}{ Percentage male (\%) } \\
\hline$\geq 60.0$ & $1.02(0.17$ to 1.87$)$ & 0.019 & - & - & 0.014 \\
\hline$<60.0$ & $-0.13(-0.53$ to 0.27$)$ & 0.527 & 6.1 & 0.302 & \\
\hline \multicolumn{6}{|l|}{ Control } \\
\hline $\mathrm{ARB}$ & $0.71(0.04$ to 1.39$)$ & 0.037 & 9.3 & 0.294 & $<0.001$ \\
\hline $\mathrm{CCB}$ & $-0.22(-0.62$ to 0.18$)$ & 0.275 & - & - & \\
\hline \multicolumn{6}{|c|}{ Follow-up duration } \\
\hline$>24$ & $0.33(-0.64$ to 1.30$)$ & 0.504 & - & - & 0.530 \\
\hline$\leq 24$ & $0.34(-0.87$ to 1.55$)$ & 0.581 & 85.1 & 0.010 & \\
\hline \multicolumn{6}{|c|}{ Baseline PWV } \\
\hline$\geq 10.0$ & $-0.13(-0.53$ to 0.27$)$ & 0.527 & 6.1 & 0.302 & 0.014 \\
\hline$<10.0$ & $1.02(0.17$ to 1.87$)$ & 0.019 & - & - & \\
\hline \multicolumn{6}{|c|}{ Study quality } \\
\hline High & $1.02(0.17$ to 1.87$)$ & 0.019 & - & - & 0.014 \\
\hline Low & $-0.13(-0.53$ to 0.27$)$ & 0.527 & 6.1 & 0.302 & \\
\hline
\end{tabular}

PWV, pulse wave velocity; ba-PWV, brachial-ankle pulse wave velocity; CCB, calcium channel blocker; CI, confidence interval; ARB, angiotensin receptor blocker or inhibitor; DBP, diastolic blood pressure; WMD, weighted mean difference. 


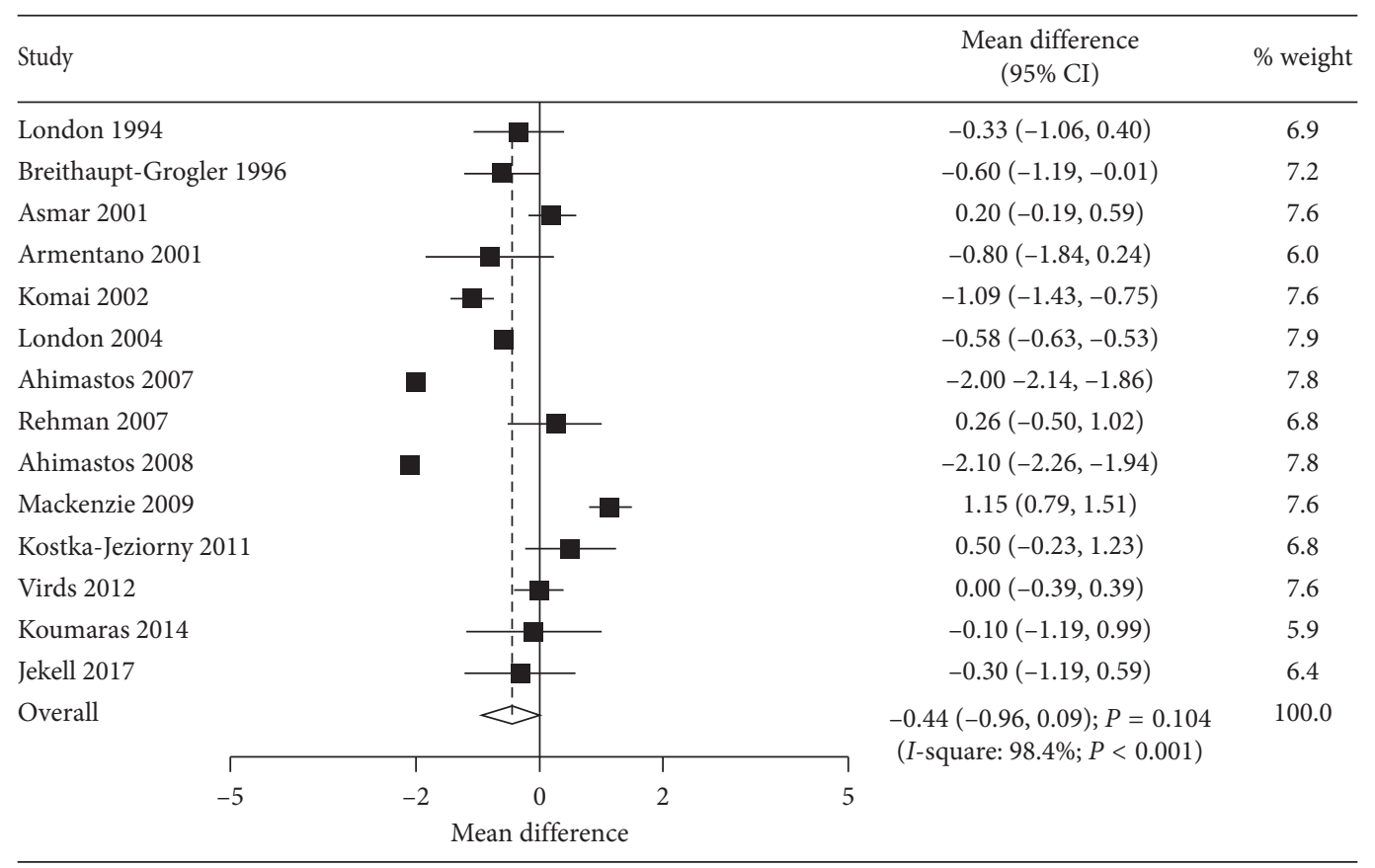

(a)

Meta-analysis estimatesm given named study is omitted

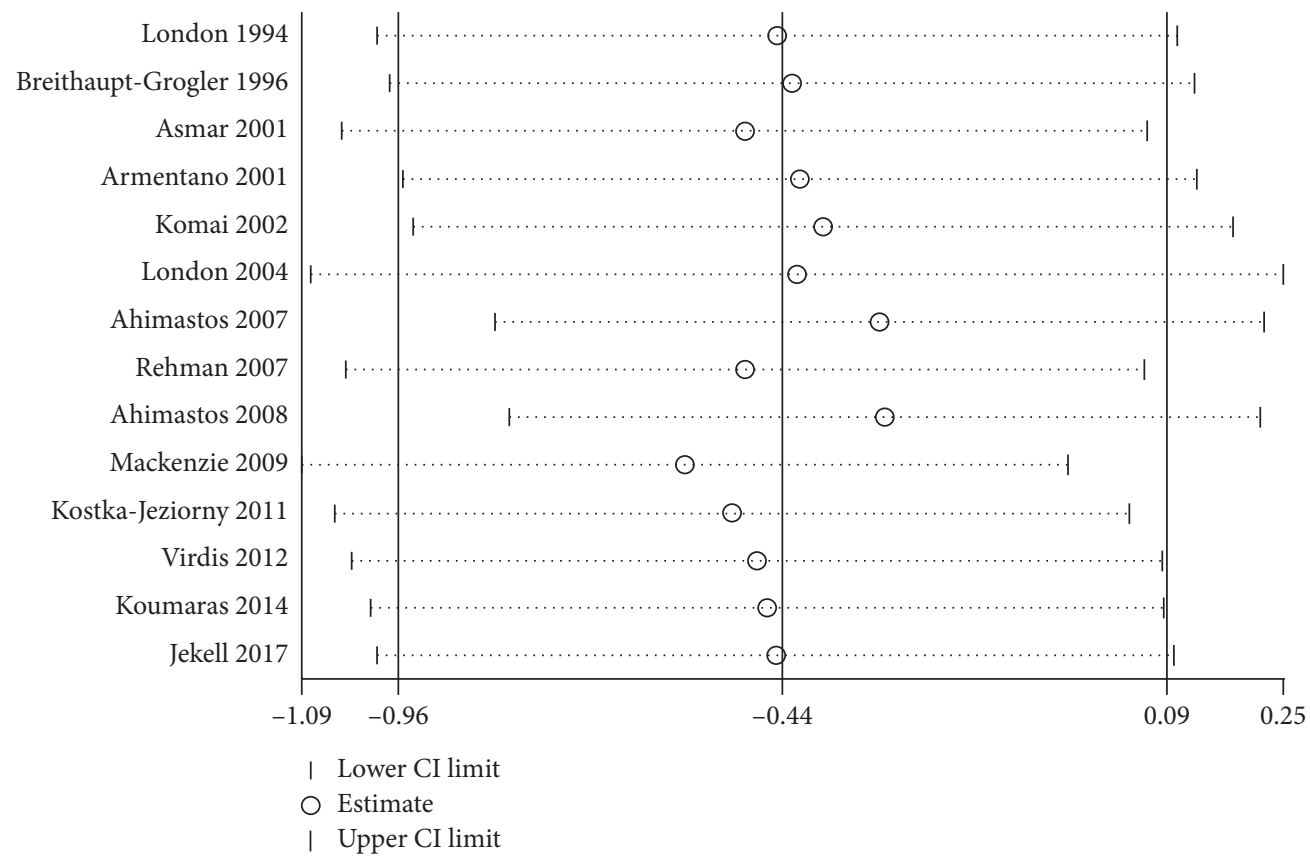

(b)

Figure 3: Continued. 


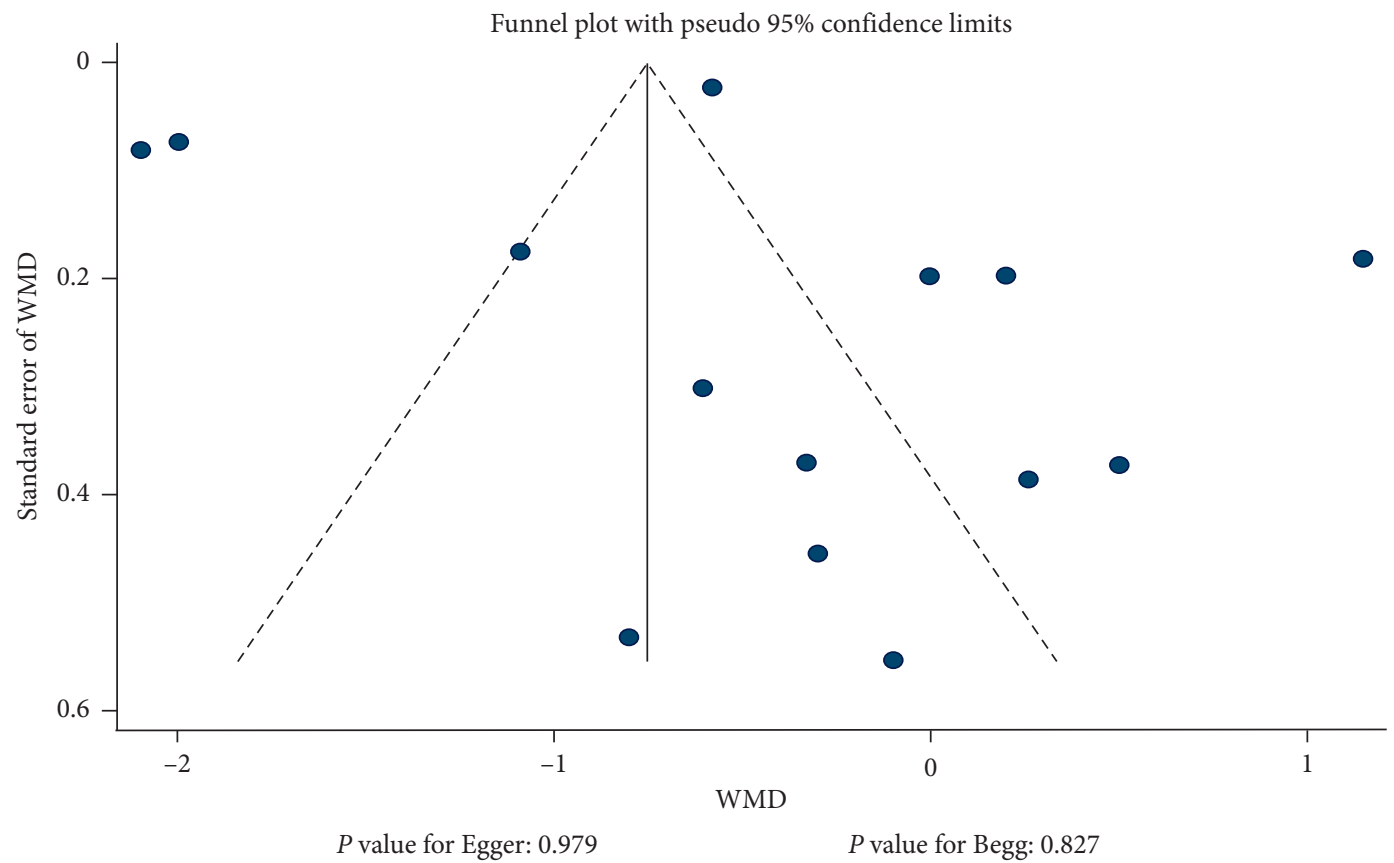

(c)

Figure 3: The results of the effect of ACEIs on cf-PWV. (a) Summary of mean differences; (b) sensitivity analysis; (c) funnel plot for publication bias assessment, by Egger's and Begg's methods. CI, confidence interval; WMD, weighted mean difference; ACEIs, angiotensin converting enzyme inhibitors; cf-PWV, carotid-femoral pulse wave velocity.

TABLE 3: Subgroup analyses for cf-PWV.

\begin{tabular}{|c|c|c|c|c|c|}
\hline Group & WMD and $95 \% \mathrm{CI}$ & $P$ value & Heterogeneity (\%) & $\begin{array}{c}P \text { value for } \\
\text { heterogeneity }\end{array}$ & $\begin{array}{c}P \text { value for } \\
\text { interaction test }\end{array}$ \\
\hline \multicolumn{6}{|l|}{ Country } \\
\hline Eastern & $-0.46(-1.78$ to 0.86$)$ & 0.496 & 90.1 & 0.001 & 0.042 \\
\hline Western & $-0.06(-0.54$ to 0.42$)$ & 0.814 & 92.5 & $<0.001$ & \\
\hline \multicolumn{6}{|c|}{ Mean age (years) } \\
\hline$\geq 60.0$ & $0.03(-2.17$ to 2.22$)$ & 0.979 & 98.7 & $<0.001$ & $<0.001$ \\
\hline$<60.0$ & $-0.18(-0.53$ to 0.17$)$ & 0.312 & 69.5 & 0.002 & \\
\hline \multicolumn{6}{|c|}{ Percentage male (\%) } \\
\hline$\geq 60.0$ & $-0.40(-0.75$ to -0.04$)$ & 0.031 & 77.5 & $<0.001$ & $<0.001$ \\
\hline$<60.0$ & $0.45(-1.00$ to 1.89$)$ & 0.547 & 92.2 & $<0.001$ & \\
\hline \multicolumn{6}{|l|}{ Control } \\
\hline ARB & $0.01(-0.31$ to 0.33$)$ & 0.961 & 0.0 & 0.642 & 0.003 \\
\hline $\mathrm{CCB}$ & $-0.33(-1.06$ to 0.40$)$ & 0.373 & - & - & \\
\hline Diuretic & $-0.07(-1.15$ to 1.01$)$ & 0.896 & 81.0 & 0.022 & \\
\hline $\mathrm{BRB}$ & $-0.18(-0.85$ to 0.48$)$ & 0.588 & 95.6 & $<0.001$ & \\
\hline \multicolumn{6}{|c|}{ Follow-up duration } \\
\hline$>24$ & $-0.25(-0.83$ to 0.33$)$ & 0.399 & 87.4 & $<0.001$ & $<0.001$ \\
\hline$\leq 24$ & $-0.10(-0.72$ to 0.53$)$ & 0.764 & 90.8 & $<0.001$ & \\
\hline \multicolumn{6}{|c|}{ Baseline PWV } \\
\hline$\geq 10.0$ & $-0.12(-0.59$ to 0.35$)$ & 0.615 & 82.7 & $<0.001$ & $<0.001$ \\
\hline$<10.0$ & $-0.15(-0.97$ to 0.67$)$ & 0.719 & 93.9 & $<0.001$ & \\
\hline \multicolumn{6}{|c|}{ Study quality } \\
\hline High & $-0.41(-0.79$ to -0.03$)$ & 0.032 & 84.9 & $<0.001$ & $<0.001$ \\
\hline Low & $0.17(-0.50$ to 0.85$)$ & 0.615 & 84.7 & $<0.001$ & \\
\hline
\end{tabular}

PWV, pulse wave velocity; cf-PWV, carotid-femoral pulse wave velocity; CCB, calcium channel blocker; $\mathrm{CI}$, confidence interval; ARB, angiotensin receptor blocker or inhibitor; DBP, diastolic blood pressure; WMD, weighted mean difference. 




(a)

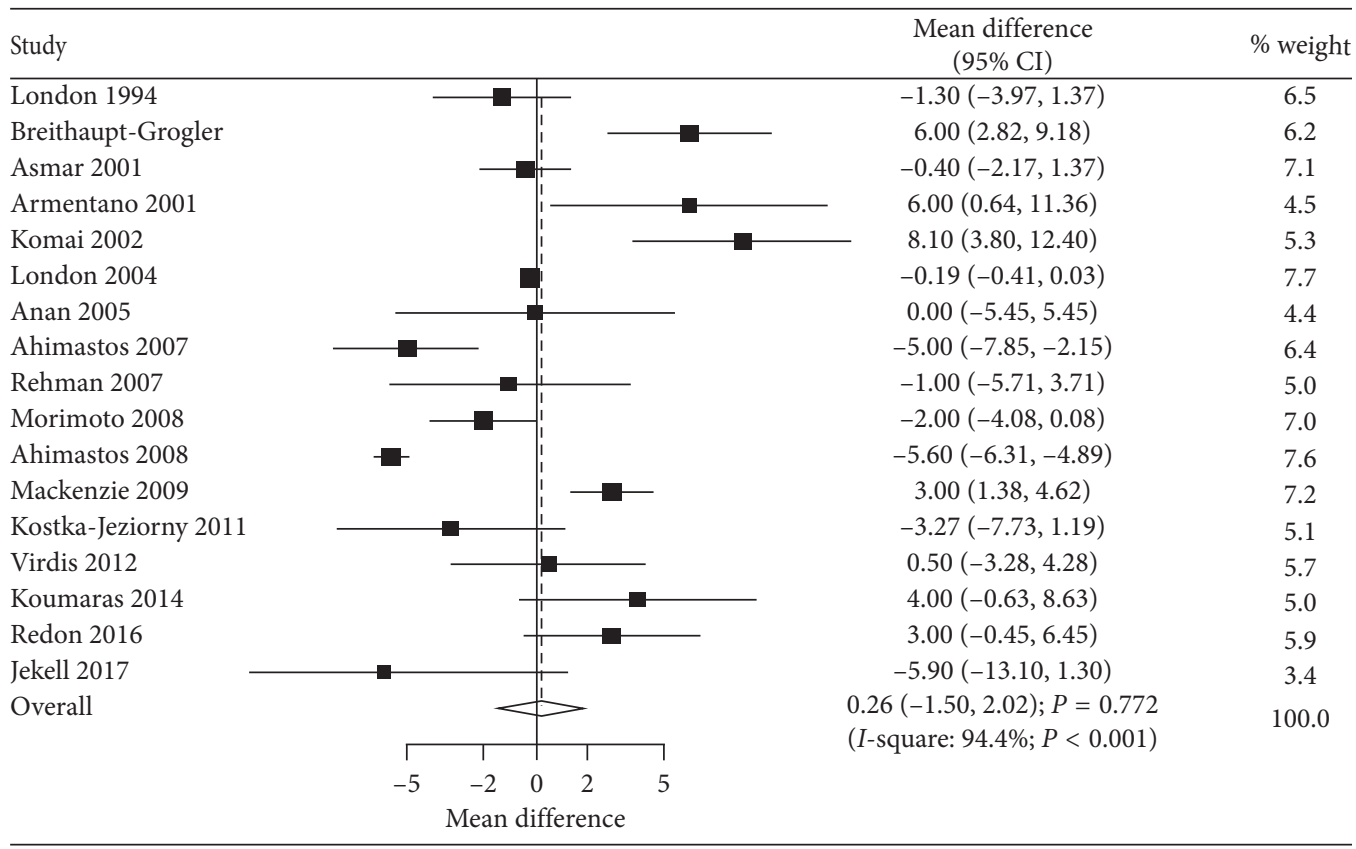

(b)

FIGURE 4: Effects of ACEIs on SBP (a) and DBP (b). Weighted mean differences are provided as well as 95\% confidence intervals (CIs). SBP, systolic blood pressure; DBP, diastolic blood pressure.

greater reduction in DBP when compared with CCB (WMD: -1.74 ; $95 \% \mathrm{CI}:-3.38$ to $-0.10 ; P=0.038)$, whereas the reduction in DBP in patients who received ACEIs was smaller when compared with BRB (WMD: 2.57 ; $95 \%$ CI: 0.39 to 4.74 ; $P=0.021$ ), and baseline PWV was <10 (WMD: 3.30; 95\% CI: 1.10 to $5.50 ; P=0.003$; Table 5).

\section{Discussion}

In this meta-analysis, the effects of ACEIs on arterial stiffness based on RCTs and changes of PWV (ba-PWV and $(f-P W V)$ were analyzed. Combined data of ba-PWV and cf-PWV levels served as a reflection of the degree of 
TABLE 4: Subgroup analyses for SBP.

\begin{tabular}{|c|c|c|c|c|c|}
\hline Group & WMD and $95 \% \mathrm{CI}$ & $P$ value & Heterogeneity (\%) & $P$ value for heterogeneity & $P$ value for interaction test \\
\hline \multicolumn{6}{|l|}{ Country } \\
\hline Eastern & $-0.05(-5.50$ to 5.40$)$ & 0.985 & 88.7 & $<0.001$ & $<0.001$ \\
\hline Western & $0.98(-3.47$ to 5.42$)$ & 0.667 & 94.6 & $<0.001$ & \\
\hline \multicolumn{6}{|c|}{ Mean age (years) } \\
\hline$\geq 60.0$ & $1.03(-3.71$ to 5.76$)$ & 0.671 & 92.1 & $<0.001$ & $<0.001$ \\
\hline$<60.0$ & $0.09(-4.72$ to 4.89$)$ & 0.972 & 90.6 & $<0.001$ & \\
\hline \multicolumn{6}{|c|}{ Percentage male (\%) } \\
\hline$\geq 60.0^{\circ}$ & $1.19(-5.17$ to 7.56$)$ & 0.713 & 96.6 & $<0.001$ & $<0.001$ \\
\hline$<60.0$ & $-0.55(-3.16$ to 2.06$)$ & 0.678 & 54.2 & 0.088 & \\
\hline \multicolumn{6}{|l|}{ Control } \\
\hline $\mathrm{ARB}$ & $-0.09(-4.90$ to 4.73$)$ & 0.972 & 66.2 & 0.019 & $<0.001$ \\
\hline $\mathrm{CCB}$ & $-1.57(-5.61$ to 2.47$)$ & 0.446 & 52.1 & 0.149 & \\
\hline Diuretic & $5.81(-10.01$ to 21.62$)$ & 0.472 & 92.8 & $<0.001$ & \\
\hline $\mathrm{BRB}$ & $0.41(-5.98$ to 6.80$)$ & 0.900 & 97.6 & $<0.001$ & \\
\hline \multicolumn{6}{|c|}{ Follow-up duration } \\
\hline$>24$ & $-4.43(-8.61$ to -0.25$)$ & 0.038 & 85.0 & $<0.001$ & $<0.001$ \\
\hline$\leq 24$ & $2.25(-0.90$ to 5.40$)$ & 0.162 & 82.7 & $<0.001$ & \\
\hline \multicolumn{6}{|c|}{ Baseline PWV } \\
\hline$\geq 10.0$ & $-2.75(-6.19$ to 0.70$)$ & 0.118 & 88. & $<0.001$ & $<0.001$ \\
\hline$<10.0$ & 3.67 (0.11 to 7.23$)$ & 0.043 & 79.2 & $<0.001$ & \\
\hline \multicolumn{6}{|c|}{ Study quality } \\
\hline High & $-0.14(-6.27$ to 6.00$)$ & 0.965 & 96.9 & $<0.001$ & $<0.001$ \\
\hline Low & $1.24(-2.06$ to 4.54$)$ & 0.461 & 75.4 & $<0.001$ & \\
\hline
\end{tabular}

ARB, angiotensin receptor blocker or inhibitor; CCB, calcium channel blocker; CI, confidence interval; PWV, pulse wave velocity; SBP, systolic blood pressure; WMD, weighted mean difference.

TABLE 5: Subgroup analyses for DBP.

\begin{tabular}{|c|c|c|c|c|c|}
\hline Group & WMD and $95 \% \mathrm{CI}$ & $P$ value & Heterogeneity (\%) & $P$ value for heterogeneity & $P$ value for interaction test \\
\hline \multicolumn{6}{|l|}{ Country } \\
\hline Eastern & $1.18(-3.54$ to 5.90$)$ & 0.624 & 82.7 & 0.001 & \multirow{2}{*}{0.910} \\
\hline Western & $1.19(-0.33$ to 2.71$)$ & 0.125 & 78.0 & $<0.001$ & \\
\hline \multicolumn{6}{|c|}{ Mean age (years) } \\
\hline$\geq 60.0$ & $2.70(-1.98$ to 7.39$)$ & 0.258 & 91.4 & $<0.001$ & \multirow[t]{2}{*}{0.004} \\
\hline$<60.0$ & $0.24(-1.21$ to 1.69$)$ & 0.744 & 46.1 & 0.054 & \\
\hline \multicolumn{6}{|c|}{ Percentage male (\%) } \\
\hline$\geq 60.0$ & $1.69(-0.80$ to 4.19$)$ & 0.184 & 76.7 & $<0.001$ & \multirow[t]{2}{*}{0.162} \\
\hline$<60.0$ & $-0.00(-2.96$ to 2.95$)$ & 0.998 & 81.8 & 0.001 & \\
\hline \multicolumn{6}{|l|}{ Control } \\
\hline ARB & $0.25(-2.15$ to 2.65$)$ & 0.839 & 26.8 & 0.243 & \multirow[t]{4}{*}{0.026} \\
\hline $\mathrm{CCB}$ & $-1.74(-3.38$ to -0.10$)$ & 0.038 & 0.0 & 0.685 & \\
\hline Diuretic & $1.50(-7.58$ to 10.58$)$ & 0.746 & 70.9 & 0.001 & \\
\hline $\mathrm{BRB}$ & $2.57(0.39$ to 4.74$)$ & 0.021 & 86.4 & $<0.001$ & \\
\hline \multicolumn{6}{|c|}{ Follow-up duration } \\
\hline$>24$ & $-0.20(-0.42$ to 0.02$)$ & 0.074 & 0.0 & 0.870 & \multirow[t]{2}{*}{$<0.001$} \\
\hline$\leq 24$ & $1.89(-0.34$ to 4.12$)$ & 0.096 & 77.7 & $<0.001$ & \\
\hline \multicolumn{6}{|c|}{ Baseline PWV } \\
\hline$\geq 10.0$ & $-0.58(-1.55$ to 0.40$)$ & 0.249 & 34.2 & 0.155 & \multirow{2}{*}{$<0.001$} \\
\hline$<10.0$ & $3.30(1.10$ to 5.50$)$ & 0.003 & 62.9 & 0.013 & \\
\hline \multicolumn{6}{|c|}{ Study quality } \\
\hline High & $1.51(-0.53$ to 3.55$)$ & 0.146 & 76.2 & $<0.001$ & \multirow[t]{2}{*}{0.020} \\
\hline Low & $0.75(-1.60$ to 3.11$)$ & 0.530 & 78.2 & $<0.001$ & \\
\hline
\end{tabular}

$\mathrm{ARB}$, angiotensin receptor blocker or inhibitor; $\mathrm{CCB}$, calcium channel blocker; CI, confidence interval; PWV, pulse wave velocity; DBP, diastolic blood pressure; WMD, weighted mean difference.

atherosclerosis in the upper-limb and lower-limb indicators. The results of our study showed no significant differences between ACEIs and control in improving the vascular stiffness, including ba-PWV and cf-PWV. Subgroup analyses indicated that the therapeutic effects of ACEIs on ba-PWV could affect by country, mean age, 
percentage male, control, baseline PWV, and study quality, while cf-PWV levels might be affected by country, mean age, percentage male, control, follow-up duration, baseline PWV, and study quality. Moreover, BP reduction in patients who received ACEIs and controls showed no significant difference.

Numerous meta-analyses have investigated the impact of ACEIs on arterial stiffness [17, 28, 57]. Mallareddy et al. [28] performed a meta-analysis to investigate the impact of ACEIs on PWV or augmentation index. The results revealed that reduction in PWV was $-1.15 \mathrm{~m} / \mathrm{s}$ for cf-PWV and $-1.9 \mathrm{~m} / \mathrm{s}$ for ba-PWV for patients who received ACEIs. Furthermore, they pointed out that ACEIs had modest beneficial influence on arterial stiffness, which was partly independent of the changes in BP. [28] Shahin et al. examined the effects of ACEIs on arterial stiffness and wave reflections in patients with hypertension. The results revealed that ACEIs vs. placebo showed significantly reduced levels of PWV, while comparison with other antihypertensive drugs showed no significant differences in PWV levels. Moreover, ACEIs reduced PWV levels in patients with different pathological conditions [57]. Xie et al. conducted a meta-analysis based on 8 RCTs and concluded no significant differences between ACEIs and atenolol on the levels of ba-PWV and cf-PWV. Also, ACEIs were inferior over atenolol in peripheral DBP and heart rate, while the levels of peripheral SBP between ACEIs and atenolol showed no significant difference [17]. However, there are several limitations in the previous meta-analyses studies that should be mentioned: (1) various designs of included studies might affect the therapeutic effects of ACEIs on arterial stiffness; (2) the studies included designs of cross-over trial, and had different washout periods, which in turn could bias the effectiveness of ACEIs; (3) the summary results of PWV were combined, and the type of ba-PWV and cf-PWV was analyzed, respectively; and (4) the therapeutic effects of ACEIs on arterial stiffness in patients with specific characteristics were not illustrated. Therefore, this meta-analysis was conducted based on RCTs to minimize the abovementioned limitations to ensure the confidence of our results.

From the funnel plots of the above two analyses, the study using PWV as detection index was less likely to be biased. Therefore, the conclusions of this study will be able to reflect changes in vascular function. Furthermore, the results of this study showed no significant differences between ACEIs and control for the indexes of arterial stiffness. The possible reason for this could be due to the use of various antihypertensive drugs, and the net therapeutic effects of ACEIs among the included trials varied. Moreover, the summary results and 95\% CIs of ba-PWV and cf-PWV were affected by the WMD of individual trial. In addition, the treatment effects of ACEI on arterial stiffness could affect the effect size of BP changes. This study showed no significant differences between ACEIs and control regarding the changes of SBP and DBP, suggesting that the therapeutic effects of ACEIs on arterial stiffness were not biased by the changes of SBP and DBP.

The significant therapeutic effects of ACEIs were observed for ba-PWV when compared with ARB or placebo (Table 2). These results indicated that ARB provided superior effects on ba-PWV than ACEIs. Also, ACEIs showed greater reduction in ba-PWV when compared with placebo (Table 2). In addition, the therapeutic effects of ACEIs on cfPWV levels were affected by country, mean age, percentage male, control, follow-up duration, baseline PWV, and study quality (Table 3). Firstly, the background therapies and lifestyles were correlated with country and mean age, and were associated with the therapeutic effects of ACEI; secondly, the differences in risk stratification between men and women could affect the levels of arterial stiffness; thirdly, the type of control drugs was correlated with the net therapeutic effects between the intervention and control groups; fourthly, the baseline PWV level was associated with the severity of arterial stiffness; and the study quality was correlated with the evidence level and reliability of summary results. However, the results of subgroup analyses were considered unreliable due to the stability of pooled results and substantial heterogeneity within the subgroups.

Although the study was professionally conducted, there were still a few shortcomings that need to be noted. (1) The purpose of this study was to analyze the curative effects of ACEIs on hypertensive patients by meta-analysis. Some studies did not mention the basis for estimating the sample size or did not fully implement the blinding method, and the study sample size was not large enough, impacting on the test results to a certain extent. (2) There were specific differences in the clinical design, type of drug, dose, follow-up time, etc. The usage of ACEIs varied between the included studies, as well as administration of drugs and the duration of intervention, contributing to the heterogeneity of subgroup analysis of the reported endpoints. In addition, other antihypertensive drugs were used as controls. (3) The relatively small number of included studies when combined with large sample clinical trials and small sample size test may lead to bias.

\section{Conclusions}

Taken together, although there are some limitations, the strengths and inferences of the overall conclusion are overwhelming. Meta-analysis of RCTs suggested that the role of ACEIs in improving arterial stiffness in hypertensive patients were observed in several subsets. These conclusions may not be applicable to the overall populations, and more evidences are needed to compare the prognosis of patients taking ACEIs with other drugs. Future large-scale studies to verify the results of subgroup analyses in this meta-analysis should be conducted.

\section{Conflicts of Interest}

The author(s) declare(s) that there is no conflict of interest regarding the publication of this paper.

\section{Acknowledgments}

This study was supported by grants from the National Natural Science Foundation of China [grant numbers NSFC-81670385/81270332], Lanzhou Talent Innovation and Entrepreneurship Project [grant numbers 2016-RC-54 and 
2016-RC-104], and the Foundation of Lanzhou University Second Hospital [grant numbers ynbskyjj 2015-2-1].

\section{References}

[1] M. Ecobici and C. Stoicescu, "Arterial stiffness and hypertension-which comes first?," Maedica (Buchar), vol. 12, no. 3, pp. 184-190, 2017.

[2] H. C. S. Muela, V. A. Costa-Hong, M. S. Yassuda et al., "Higher arterial stiffness is associated with lower cognitive performance in patients with hypertension," The Journal of Clinical Hypertension, vol. 20, no. 1, pp. 22-30, 2018.

[3] M. E. Safar, "Arterial stiffness as a risk factor for clinical hypertension," Nature Reviews Cardiology, vol. 15, no. 2, pp. 97-105, 2018.

[4] H. Tomiyama, K. Shiina, C. Matsumoto-Nakano et al., "The contribution of inflammation to the development of hypertension mediated by increased arterial stiffness," Journal of the American Heart Association, vol. 6, no. 7, 2017.

[5] Y. Chen, J. H. Liu, Z. Zhen et al., "Assessment of left ventricular function and peripheral vascular arterial stiffness in patients with dipper and non-dipper hypertension," Journal of Investigative Medicine, vol. 66, no. 2, pp. 319-324, 2017.

[6] S. Chang, J. Kim, T. Sohn, H. Son, and J. Lee, "Effects of glucose control on arterial stiffness in patients with type 2 diabetes mellitus and hypertension: an observational study," Journal of International Medical Research, vol. 46, no. 1, pp. 284-292, 2017.

[7] K. Uneda, K. Tamura, H. Wakui et al., "Comparison of direct renin inhibitor and angiotensin II receptor blocker on clinic and ambulatory blood pressure profiles in hypertension with chronic kidney disease," Clinical and Experimental Hypertension, vol. 38, no. 8, pp. 738-743, 2016.

[8] G. C. Oh, H. Y. Lee, W. J. Chung et al., "Comparison of effects between calcium channel blocker and diuretics in combination with angiotensin II receptor blocker on 24-h csentral blood pressure and vascular hemodynamic parameters in hypertensive patients: study design for a multicenter, doubleblinded, active-controlled, phase 4, randomized trial," Clinical Hypertension, vol. 23, no. 1, 2017.

[9] C. Chu, Y. Dai, J. Mu et al., "Associations of risk factors in childhood with arterial stiffness 26 years later," Journal of Hypertension, vol. 35, no. 1, pp. S10-S15, 2017.

[10] J. Nemcsik, O. Cseprekál, and A. Tislér, "Measurement of arterial stiffness: a novel tool of risk stratification in hypertension," Advances in Experimental Medicine and Biology, vol. 956, pp. 475-488, 2017.

[11] J. Barochiner, L. S. Aparicio, J. Alfie et al., "Arterial stiffness in treated hypertensive patients with white-coat hypertension," The Journal of Clinical Hypertension, vol. 19, no. 1, pp. 6-10, 2017.

[12] A. C. Cameron, N. N. Lang, and R. M. Touyz, "Drug treatment of hypertension: focus on vascular health," Drugs, vol. 76, no. 16, pp. 1529-1550, 2016.

[13] M. E. Safar, "Blood pressure variability and arterial stiffness," Artery Research, vol. 5, no. 4, pp. 119-121, 2011.

[14] T. Miyoshi, T. Murakami, S. Sakuragi et al., "Comparable effect of aliskiren or a diuretic added on an angiotensin II receptor blocker on augmentation index in hypertension: a multicentre, prospective, randomised study," Open heart, vol. 4, no. 1, 2017.

[15] M. Volpe, "Should all patients at high cardiovascular risk receive renin-angiotensin system blockers?," QJM, vol. 105, no. 1, pp. 11-27, 2012.
[16] M. Ahluwalia and S. Bangalore, "Management of hypertension in 2017," Current Opinion in Cardiology, vol. 32, no. 4, pp. 413-421, 2017.

[17] H. Xie, G. Luo, Y. Zheng, F. Peng, and L. Xie, "A meta-analytical comparison of atenolol with angiotensin-converting enzyme inhibitors on arterial stiffness, peripheral blood pressure and heart rate in hypertensive patients," Clinical and Experimental Hypertension, vol. 39, no. 5, pp. 421-426, 2017.

[18] F. Sun, Y. Song, J. Liu et al., "Efficacy of losartan for improving insulin resistance and vascular remodeling in hemodialysis patients," Hemodialysis International, vol. 20, no. 1, pp. 22-30, 2016.

[19] M. Volpe and G. Tocci, "Olmesartan in the treatment of hypertension in elderly patients: a review of the primary evidence," Drugs \& Aging, vol. 30, no. 12, pp. 987-998, 2013.

[20] A. R. Zankl, B. Ivandic, M. Andrassy et al., "Telmisartan improves absolute walking distance and endothelial function in patients with peripheral artery disease," Clinical Research in Cardiology, vol. 99, no. 12, pp. 787-794, 2010.

[21] E. A. Andreadis, M. E. Sfakianakis, G. I. Tsourous et al., "Differential impact of angiotensin receptor blockers and calcium channel blockers on arterial stiffness," International angiology, vol. 29, no. 3, pp. 266-272, 2010.

[22] W. Niu and Y. Qi, "A meta-analysis of randomized controlled trials assessing the impact of beta-blockers on arterial stiffness, peripheral blood pressure and heart rate," International Journal of Cardiology, vol. 218, pp. 109-117, 2016.

[23] U. Raff, S. Walker, C. Ott, M. P. Schneider, and R. E. Schmieder, "Olmesartan improves pulse wave velocity and lowers central systolic blood pressure and ambulatory blood pressure in patients with metabolic syndrome," The Journal of Clinical Hypertension, vol. 17, no. 2, pp. 98-104, 2015.

[24] G. Mancia, G. de Backer, A. Dominiczak et al., "2013 guidelines for the management of arterial hypertension: the task force for the management of arterial hypertension of the European society of hypertension (ESH) and of the European society of Cardiology (ESC)," European Heart Journal, vol. 34, no. 28, pp. 2159-2219, 2013.

[25] L. Boesby, T. Elung-Jensen, S. Strandgaard, and A. L. Kamper, "Eplerenone attenuates pulse wave reflection in chronic kidney disease stage 3-4-a randomized controlled study," PLoS One, vol. 8, no. 5, Article ID e64549, 2013.

[26] D. Hayoz, D. H. Zappe, M. A. R. Meyer et al., "Changes in aortic pulse wave velocity in hypertensive postmenopausal women: comparison between a calcium channel blocker vs. angiotensin receptor blocker regimen," The Journal of Clinical Hypertension, vol. 14, no. 11, pp. 773-778, 2012.

[27] E. J. Kim, W.-H. Song, J. U. Lee et al., "Efficacy of losartan and carvedilol on central hemodynamics in hypertensives: a prospective, randomized, open, blinded end point, multicenter study," Hypertension Research, vol. 37, no. 1, pp. 50-56, 2014.

[28] M. Mallareddy, C. R. Parikh, and A. J. Peixoto, "Effect of angiotensin-converting enzyme inhibitors on arterial stiffness in hypertension: systematic review and meta-analysis," The Journal of Clinical Hypertension, vol. 8, no. 6, pp. 398-403, 2006.

[29] P. A. Kithas and M. A. Supiano, "Spironolactone and hydrochlorothiazide decrease vascular stiffness and blood pressure in geriatric hypertension," Journal of the American Geriatrics Society, vol. 58, no. 7, pp. 1327-1332, 2010.

[30] D. Moher, A. Liberati, J. Tetzlaff, D. G. Altman, and P. Group, "Preferred reporting items for systematic reviews and meta- 
analyses: the PRISMA statement," PLoS Medicine, vol. 6, no. 7, Article ID e1000097, 2009.

[31] A. R. Jadad, R. A. Moore, D. Carroll et al., "Assessing the quality of reports of randomized clinical trials: is blinding necessary?," Controlled Clinical Trials, vol. 17, no. 1, pp. 1-12, 1996.

[32] R. DerSimonian and N. Laird, "Meta-analysis in clinical trials," Controlled Clinical Trials, vol. 7, no. 3, pp. 177-188, 1986.

[33] A. E. Ades, G. Lu, and J. P. T. Higgins, "The interpretation of random-effects meta-analysis in decision models," Medical Decision Making, vol. 25, no. 6, pp. 646-654, 2005.

[34] J. J. Deeks, J. P. T. Higgins, and D. G. Altman, “Analyzing data and undertaking meta-analyses," in Cochrane Handbook for Systematic Reviews of Interventions 501, J. Higgins and S. Green, Eds., pp. 243-296, The Cochrane Collaboration, Oxford, UK, 2008.

[35] J. P. T. Higgins, S. G. Thompson, J. J. Deeks, and D. G. Altman, "Measuring inconsistency in meta-analyses," BMJ, vol. 327, no. 7414, pp. 557-560, 2003.

[36] A. Tobias, "Assessing the influence of a single study in metaanalysis,” Stata Technical Bulletin, vol. 47, pp. 15-17, 1999.

[37] D. G. Altman and J. M. Bland, "Statistics Notes: interaction revisited: the difference between two estimates," BMJ, vol. 326, no. 7382, p. 219, 2003.

[38] M. Egger, G. D. Smith, M. Schneider, and C. Minder, "Bias in meta-analysis detected by a simple, graphical test," BMJ, vol. 315, no. 7109, pp. 629-634, 1997.

[39] C. B. Begg and M. Mazumdar, "Operating characteristics of a rank correlation test for publication bias," Biometrics, vol. 50, no. 4, pp. 1088-1101, 1994.

[40] G. M. London, B. Pannier, A. P. Guerin, S. J. Marchais, M. E. Safar, and J. L. Cuche, "Cardiac hypertrophy, aortic compliance, peripheral resistance, and wave reflection in endstage renal disease. Comparative effects of ACE inhibition and calcium channel blockade," Circulation, vol. 90, no. 6, pp. 2786-2796, 1994.

[41] K. Breithaupt-Grögler, M. Leschinger, G. G. Belz et al., "Influence of antihypertensive therapy with cilazapril and hydrochlorothiazide on the stiffness of the aorta," Cardiovascular Drugs and Therapy, vol. 10, no. 1, pp. 49-57, 1996.

[42] R. G. Asmar, G. M. London, M. E. O'Rourke, and M. E. Safar, "Improvement in blood pressure, arterial stiffness and wave reflections with a very-low-dose perindopril/indapamide combination in hypertensive patient," Hypertension, vol. 38, no. 4, pp. 922-926, 2001.

[43] R. L. Armentano, S. Graf, A. J. Ramirez et al., "Mechanical vs. intrinsic components in the improvement of brachial arterial compliance. Comparison of the effects of atenolol versus ramipril in hypertensive patients," Medicina (B Aires), vol. 61, pp. 535-540, 2001.

[44] N. Komai, M. Ohishi, R. Morishita et al., "Serum hepatocyte growth factor concentration is correlated with the forearm vasodilator response in hypertensive patients," American Journal of Hypertension, vol. 15, no. 6, pp. 499-506, 2002.

[45] G. M. London, R. G. Asmar, M. F. O’Rourke, M. E. Safar, and R. P. Investigators, "Mechanism(s) of selective systolic blood pressure reduction after a low-dose combination of perindopril/indapamide in hypertensive subjects: comparison with atenolol," Journal of the American College of Cardiology, vol. 43, no. 1, pp. 92-99, 2004.

[46] F. Anan, N. Takahashi, T. Ooie et al., "Effects of valsartan and perindopril combination therapy on left ventricular hypertrophy and aortic arterial stiffness in patients with essential hypertension," European Journal of Clinical Pharmacology, vol. 61 , no. 5-6, pp. 353-359, 2005.

[47] A. A. Ahimastos, A. Aggarwal, K. M. D’Orsa et al., "Effect of perindopril on large artery stiffness and aortic root diameter in patients with marfan syndrome," JAMA, vol. 298, no. 13, pp. 1539-1547, 2007.

[48] A. Rehman, S. Ismail, L. Naing, T. Roshan, and A. Rahman, "Reduction in arterial stiffness with angiotensin II antagonism and converting enzyme inhibition: a comparative study among Malay hypertensive subjects with a known genetic profile," American Journal of Hypertension, vol. 20, no. 2, pp. 184-189, 2007.

[49] S. Morimoto, K. Maki, Y. Aota, T. Sakuma, and T. Iwasaka, "Beneficial effects of combination therapy with angiotensin II receptor blocker and angiotensin-converting enzyme inhibitor on vascular endothelial function," Hypertension Research, vol. 31, no. 8, pp. 1603-1610, 2008.

[50] A. A. Ahimastos, A. M. Dart, A. Lawler, P. A. Blombery, and B. A. Kingwell, "Reduced arterial stiffness may contribute to angiotensin-converting enzyme inhibitor induced improvements in walking time in peripheral arterial disease patients," Journal of Hypertension, vol. 26, no. 5, pp. 1037-1042, 2008.

[51] I. S. Mackenzie, C. M. McEniery, Z. Dhakam, M. J. Brown, J. R. Cockcroft, and I. B. Wilkinson, "Comparison of the effects of antihypertensive agents on central blood pressure and arterial stiffness in isolated systolic hypertension," $\mathrm{Hy}$ pertension, vol. 54, no. 2, pp. 409-413, 2009.

[52] K. Kostka-Jeziorny, P. Uruski, and A. Tykarski, "Effect of allopurinol on blood pressure and aortic compliance in hypertensive patients," Blood Pressure, vol. 20, no. 2, pp. 104110, 2011.

[53] A. Virdis, L. Ghiadoni, A. A. Qasem et al., "Effect of aliskiren treatment on endothelium-dependent vasodilation and aortic stiffness in essential hypertensive patients," European Heart Journal, vol. 33, no. 12, pp. 1530-1538, 2012.

[54] C. Koumaras, K. Tziomalos, E. Stavrinou et al., "Effects of renin-angiotensin-aldosterone system inhibitors and betablockers on markers of arterial stiffness," Journal of the American Society of Hypertension, vol. 8, no. 2, pp. 74-82, 2014.

[55] J. Redon and G. Pichler, "Comparative study of the efficacy of olmesartan/amlodipine vs. Perindopril/amlodipine in peripheral and central blood pressure parameters after missed dose in type 2 diabetes," American Journal of Hypertension, vol. 29, no. 9, pp. 1055-1062, 2016.

[56] A. Jekell, M. Kalani, and T. Kahan, "The effects of alpha 1adrenoceptor blockade and angiotensin converting enzyme inhibition on central and brachial blood pressure and vascular reactivity: the doxazosin-ramipril study," Heart and Vessels, vol. 32, no. 6, pp. 674-684, 2017.

[57] Y. Shahin, J. A. Khan, and I. Chetter, "Angiotensin converting enzyme inhibitors effect on arterial stiffness and wave reflections: a meta-analysis and meta-regression of randomised controlled trials," Atherosclerosis, vol. 221, no. 1, pp. 18-33, 2012. 